\title{
1 Exploring the metastatic role of the inhibitor of apoptosis BIRC6 in Breast
}

\section{Cancer}

3 Corresponding author: Matias Luis Pidre, Pringles 3010, Lanús, Buenos Aires, Argentina, CP 1824

$4 \quad$ mlpidre@biol.unlp.edu.ar, mobile: +54 92213646836

\section{AUTHORS}

6 Santiago M. Gómez Bergna ${ }^{1}$; Abril Marchesini' ${ }^{1}$ Leslie C. Amorós Morales ${ }^{1}$; Paula N. Arrías ${ }^{1}$; Hernán

7 G. Farina ${ }^{2}$; Víctor Romanowski ${ }^{1} ;$ M. Florencia Gottardo²*; Matias L. Pidre ${ }^{1 *}$.

$8 \quad$ *Both authors equally contributed to this work.

\section{AUTHOR AFFILIATIONS}

$10{ }^{1}$ Instituto de Biotecnología y biología molecular (IBBM-CONICET-UNLP)

$11{ }^{2}$ Center of Molecular \& Translational Oncology, Department of Science and Technology,

12 National University of Quilmes, Buenos Aires, Argentina. 
14 Abstract

15 Breast cancer is the most common cancer as well as the first cause of death by cancer in women worldwide. BIRC6 (baculoviral IAP repeat-containing protein 6) is a member of the inhibitors of apoptosis protein family thought to play an important role in the progression or chemoresistance of many cancers. The aim of the present work was to investigate the role of apoptosis inhibitor BIRC6 in breast cancer, focusing particularly on its involvement in the metastatic cascade.

We analyzed BIRC6 mRNA expression levels and Copy Number Variations (CNV) in three breast cancer databases from The Cancer Genome Atlas (TCGA) comparing clinical and molecular attributes. Genomic analysis was performed using CBioportal platform while transcriptomic studies (mRNA expression levels, correlation heatmaps, survival plots and Gene Ontology) were performed with USC Xena and R. Statistical significance was set at pvalues less than 0.05

Our analyses showed that there was a differential expression of BIRC6 in cancer samples when compared to normal samples. CNV that involve amplification and gain of BIRC6 gene were correlated with negative hormone receptor tumors, higher prognostic indexes, younger age at diagnosis and both chemotherapy and radiotherapy administration. Transcriptomic and gene-ontology analyses showed that, in conditions of high BIRC6 mRNA levels, there are differential expression patterns in apoptotic, proliferation, and metastatic pathways. In summary, our in silico analyses suggest that BIRC6 exhibits an antiapoptotic, proproliferative and an apparent pro-metastatic role and could be a relevant molecular target for treatment of Breast Cancer tumors. 


\section{Introduction}

Breast cancer $(\mathrm{BC})$ is one of the most prevalent cancers in the general population, and it's the leading cause of death for female cancer patients. This pathology presents heterogeneity in the biological behavior of tumors and a great clinical variability (1). The main cause of breast cancer-related death is the development of metastasis, which accounts for $90 \%$ of deaths. The recurrence of this disease originates in local processes on secondary organs and is associated with a poor prognosis (2). The main characteristic that differentiates a benign tumor from a malignant tumor is the invasiveness of the malignant cells. This ability to invade surrounding tissues is fundamental for metastasis. Said process comprises several steps, all of which are controlled by different cellular and environmental signals (3). Understanding metastasis is of central importance when searching for antitumor therapies since identification of potential molecular targets for treatment requires discerning the key factors involved in it. Apoptosis is a highly regulated process and its failure can result in many pathological conditions including tumor development. In mammals, programmed cell death is usually regulated by the IAP family of proteins (named after their main function, IAP: "inhibitors of apoptosis proteins") (4). IAP, which were originally isolated and characterized from baculoviral genomes, contain highly conserved protein-protein interaction motifs called baculoviral IAP repeats (BIR). Through these BIR motifs, IAP are able to associate with different caspases and prevent their activity, thereby inhibiting apoptosis. Many IAPs also contain a RING domain at their C-terminal end with E3 ubiquitin ligase activity that allows control of protein levels by ubiquitination and degradation via proteasome (5). This family of proteins plays a central role in the control of survival and programmed cell death by regulating determining factors in both the caspase activation pathway as well as the NF-KB pathway (5).

The role of IAPs in tumor progression and metastasis has been reported for several tumor types (6-9). Recently, a PanCancer transcriptomic analysis showed a key role for IAPs in tumor physiology (10). 

IAP of approximately $530 \mathrm{kDa}$ that contains a BIR domain at its $\mathrm{N}$-terminal region and an ubiquitin ligase domain at its C-terminal (UBC). Different research studies have described that BIRC6 plays a dual role as an anti-apoptotic IAP and as a chimeric E2/E3 ubiquitin ligase. BIRC6 is capable of catalyzing ubiquitination of different target proteins such as SMAC/DIABLO and Caspase-9, among others (11-13). BIRC6 not only inhibits the proapoptotic protein SMAC, but also binds to procaspase-9 and prevents its cleavage $(13,14)$. Likewise, through its BIR domain it can bind and inhibit active caspases, including caspases $3,6,7$ and $9(13-16)$.

In addition to its function as an inhibitory protein of apoptosis, BIRC6 plays both an important role in cell proliferation and as a regulator of cytokinesis (17). BIRC6 is associated with the membrane and is located in the Golgi compartments and in the vesicular system (16). BIRC6 also participates in other cellular processes such as autophagy, in which it regulates autophagosome-lysosome fusion $(18,19)$.

Different groups have demonstrated that IAP are overexpressed in several types of tumor cells, and it has been inferred that they could be related to tumorigenesis, treatment resistance, worse prognosis and oncogenesis (5,7,20-30). In particular, BIRC6 overexpression has been found in tumor tissues of gastric carcinomas (31), colorectal cancer (21), breast cancer (22), and lung cancer (24) among others. These findings postulate IAP, and particularly BIRC6, as a potential therapeutic target against different cancers, especially those that most frequently develop chemoresistance. Such is the case of BC, for which BIRC6 has not yet been completely validated as a therapeutic target. Our aim was to further evaluate whether BIRC6 may play a role in $\mathrm{BC}$ and metastasis using bioinformatic tools.

\section{Materials and methods}

\subsection{Breast Cancer and normal tissue samples}

87 We used different public datasets containing clinical, genomic and transcriptomic information from patient samples. We also used two different platforms to analyze the data. 
For these analyses, the TCGA and GTEx databases and the UCSCXena platform were

90

91

92 employed. Samples corresponding to mammary tissues were filtered and the expression of BIRC6 and other genes was evaluated in conditions of normal tissue and tissue of primary tumors.

\subsection{CNV and clinical attributes}

For these analyses, the $\mathrm{BC}$ database METABRIC $(32,33)$ and the cBioPortal platform were used. The BIRC6 gene was used as a query and the correlation of different copy number variations (CNV) with the expression of BIRC6 and several clinical attributes of interest was assessed.

\subsection{Survival plots}

The UCSCXena (34) platform and the TCGA Pan-Cancer (35-38) database were used to analyze the patients survival. To this end, samples corresponding to $\mathrm{BC}$ were filtered and the expression of BIRC6 were evaluated. In the analysis, the samples were divided into two groups: high ( $\geq 11.1)$ and low expression of BIRC6 $(<11.1)$, and survival rates were plotted.

\subsection{Transcriptomic analyses}

The UCSCXena $(34)$ and cBioPortal $(39,40)$ platforms and the TCGA GTEx, TCGA PanCancer (35-38) and Molecular Taxonomy of BC International Consortium (BC, METABRIC) databases were used to evaluate the expression of BIRC6 $(32,33)$. TCGA GTEx was selected because it is the only one that includes transcriptomic data from normal tissue from healthy volunteers and tissue from primary tumors obtained from patients with BC. The TCGA PanCancer (PANCAN) and BC (METABRIC $(32,33))$ databases were selected on the basis of the number of samples included in the datasets, and the different parameters that could be evaluated. In the case of TCGA Pan-Cancer and TCGA-Target-GTEx, normalized RNAseqV2 data was employed using RSEM quantification (41). In the case of BC (METABRIC), the mRNA expression data in z-scores relative to all samples (log microarray) carried out on the Illumina HT-12 v3 platform (Illumina_Human_WG-v3) (32) was used. 
115 For different pathway correlation analyses, the TCGA Pan-Cancer database (PANCAN) and

116 the UCSCXena and CBioPortal platforms were used. Samples corresponding to BC were

117 filtered and the expression of BIRC6 and different molecules involved in the metastatic

118 cascade pathways were evaluated. In the analysis, the samples were divided into two groups:

119 high expression of BIRC6 $(\geq 11.1)$ and low expression of BIRC6 $(<11.1)$. In addition, the 120 analysis was performed for different types of BC: hormone receptor positive and hormone receptor negative. The cut-off points to consider HR positive were the following: ESR1 $\geq 10$ and PGR $\geq 7$ for RSEM normalized expression.

\subsection{Gene Ontology and pathway analysis}

124 Publicly available data corresponding to the TCGA-BC dataset was used to perform differential gene expression analysis and Gene Ontology. BC harmonized data (hg38) in HTseq- Counts (raw counts) format was downloaded from the TCGA database (https://portal.gdc.cancer.gov/) using the GDCdownload function of the TCGABiolinks package (2.18.0) (42-44) in R (45). The dataset contained raw, fully sequenced transcriptome data from 1,088 primary tumor samples from BC patients.

To perform the differential expression analysis of genes, the samples were separated into two groups: those that presented BIRC6 expression values greater than the median and those that presented BIRC6 expression values lower than the median. All samples were normalized and filtered using R / Bioconductor's TCGABiolinks package following the standard pipeline.

134 They were preprocessed using the TCGAanalyze_Preprocessing function and a correlation cutoff of 0.6, then normalized with TCGAanalyze_Normalization using the GC content method, and finally filtered using TCGAanalyze_Filtering by quantile as recommended.

137 For the enrichment analysis, the TCGAanalyze_EAcomplete function was applied using the DEGs (Differential Expressed Gene) with a $\log (\mathrm{FC})>0$ for overexpressed DEGs or $\log (\mathrm{FC})<0$ for less expressed DEGs, in order to obtain the 3 ontologies of those genes, respectively (GO: biological process, GO: cellular component, and GO: molecular function) and the pathways in 
141 which they were involved. These results were plotted using the TCGAvisualize_EAbarplot

142 function, showing the 35 biological processes with the lowest FDR.

\section{$143 \quad 2.6$ Statistical analysis}

144 Expression of BIRC6 in samples of healthy volunteers and samples of mammary tumor tissue: $\mathrm{n}=1275$ ( $\mathrm{n}=1099$ primary tumors; $\mathrm{n}=176$ normal breast tissue); $\mathrm{t}$-Test. Expression of BIRC6 vs. copy number: $\mathrm{n}=2173$ ( $\mathrm{n}=232$ Deletions; $\mathrm{n}=1818$ Diploids; $\mathrm{n}=111$ Gains; $\mathrm{n}=12$

147 Amplifications); multiple ANOVA followed by TukeyHSD. BIRC6 CNV vs. presence / absence

148 of receptors: $n=2140$ was used to evaluate the estrogen receptor $(n=1617$ ER $+; n=523$

149 ER-), $n=1980$ to evaluate the progesterone receptor $(n=1040 P R+; n=940 P R-)$ and $n=$ 1980 to evaluate the HER2 receptor ( $n=247$ HER2 +; $n=1733$ HER2 -); X2 test grouping CNV that implied an increase in the number of copies (gains and amplifications) and those that did not imply it (deletions and diploids). BIRC6 CNV vs. age at diagnosis and the Nottingham Prognostic Index: $\mathrm{n}=2173$ ( $\mathrm{n}=232$ Deletions; $\mathrm{n}=1818$ Diploids; $\mathrm{n}=111$ Gains; $\mathrm{n}=12$ Amplifications); multiple ANOVA followed by TukeyHSD. BIRC6 CNV vs. Neoplastic Histological Grade: $\mathrm{n}=2072(\mathrm{n}=174$ Grade 1; $\mathrm{n}=851$ Grade 2; $\mathrm{n}=1047$ Grade 3); $\mathrm{x} 2$ test grouping CNV that implied an increase in the number of copies of the gene (gains and amplifications) and those that did not imply it (deletions and diploids). BIRC6 CNV vs. treatment with chemotherapy or radiotherapy: $n=1980(n=1173$ Treated; $n=807$ Not Treated $)$ in the case of radiotherapy and $n=1980(n=411$ Treated; $n=1569$ Untreated $)$ in the case of chemotherapy; $x 2$ test by grouping those CNV that implied an increase in the number of copies (gains and amplifications) and those that did not imply it (deletions and diploids). BIRC6 expression vs. proteins of different pathways: $n=1211(\mathrm{n}=697$ High exceed 0.05 for all studies. 


\section{Results}

\subsection{Patient cohort}

For clinical, genomic and transcriptomic analyses three different databases were employed.

Patient attributes of each database are summarized in Table 1. The numbers in each cell indicate the number of patients with the corresponding attribute.

\begin{tabular}{|c|c|c|c|c|}
\hline \multirow{2}{*}{\multicolumn{2}{|c|}{ Clinical attribute }} & \multicolumn{3}{|c|}{ Database } \\
\hline & & METABRIC & TCGA, PanCancer & TCGA-BRCA 2021 \\
\hline \multicolumn{2}{|c|}{ Number of patients (BC) } & 2509 & 1084 & 1098 \\
\hline \multirow{2}{*}{ Age } & $\leq 50$ & 567 & 294 & 297 \\
\hline & $>50$ & 1926 & 756 & 766 \\
\hline Histological & 1 & 214 & Not available & Not available \\
\hline \multirow{2}{*}{ Grade } & 2 & 976 & Not available & Not available \\
\hline & 3 & 1198 & Not available & Not available \\
\hline \multirow{2}{*}{ ER Status } & Positive & 1825 & Not available & Not available \\
\hline & Negative & 644 & Not available & Not available \\
\hline \multirow{2}{*}{ PR Status } & Positive & 1040 & Not available & Not available \\
\hline & Negative & 940 & Not available & Not available \\
\hline \multirow{2}{*}{ HER2 Status } & Positive & 247 & Not available & Not available \\
\hline & Negative & 1733 & Not available & Not available \\
\hline \multirow{2}{*}{ Hormone Therapy } & Yes & 1216 & Not available & Not available \\
\hline & No & 764 & Not available & Not available \\
\hline \multirow{2}{*}{ Chemotherapy } & Yes & 412 & Not available & 1097 \\
\hline & No & 1568 & Not available & 1097 \\
\hline \multirow{2}{*}{ Radiotherapy } & Yes & 1173 & 549 & 1097 \\
\hline & No & 807 & 434 & 1097 \\
\hline
\end{tabular}

171 Table 1. Patient cohort. Table 1 shows principal clinical attributes corresponding to each of three

172 databases used in this work. The number in each cell indicates the quantity of patients with clinical 173 attributes specified in column one. 


\section{$174 \quad 3.2$ BIRC6 is differentially expressed in tumor samples}

175 In order to evaluate the role of BIRC6 in human BC samples, we proceeded to study

176 transcriptomic databases.

177 The expression of BIRC6 was compared in samples from primary tumors of BC patients and

178 normal tissue samples from healthy volunteers. The result is shown in Fig 1A. We observed a

179 statistically significant increase in the expression of BIRC6 in primary tumor samples in 180 comparison to normal tissue from healthy volunteers. Cancer patients had a median z-score 181 of 0.113 , whilst normal tissue has a median z-score of -0.372 .

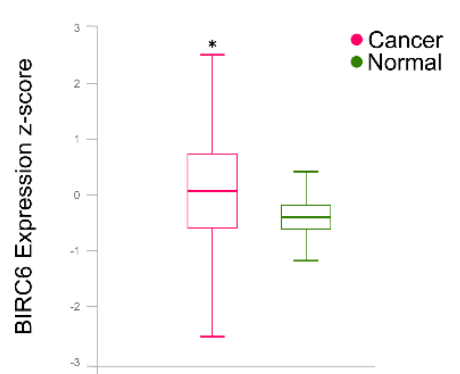

C

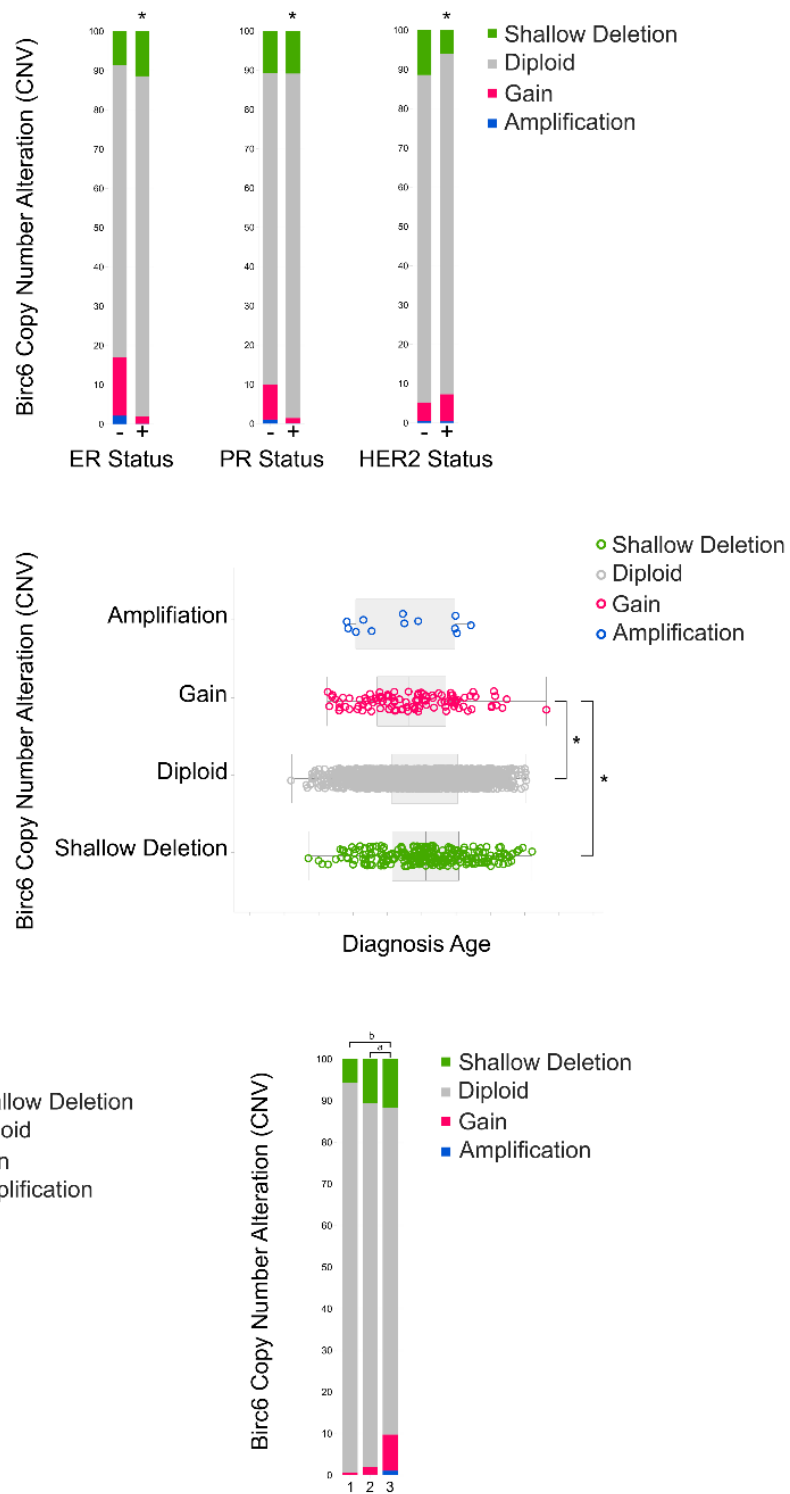

Neoplasm Histologic Grade 
Fig 1. BIRC6 expression and copy number variation (CNV). (A) Differential expression of BIRC6 between tumor and normal tissue. (B) BIRC6 CNV abundance vs. different clinical attributes: ER, PR and HER2 status, $x 2{ }^{*} p<0.05$; (C) chemotherapy and radiotherapy, $x 2{ }^{*} p<0.05$; (D) diagnosis age, multiple ANOVA followed by TukeyHSD * $\mathrm{p}<0.05$; (E) Notingham prognostic index, multiple ANOVA followed by TukeyHSD * $p<0.05$ and neoplasm histologic grade, $x 2{ }^{*} p<0.05$.

3.3 Increased copy number of the BIRC6 gene correlates with higher cellular dedifferentiation and worse prognosis

To characterize whether the alteration of BIRC6 has an impact on the different clinical attributes of patients we first evaluated if BIRC6 gene copy number variation (CNV) implied any change of its expression level. The CNV data was divided into four groups: amplifications, gains, diploids and deletions. A direct relationship was found between copy number and mRNA expression. Samples with gene amplifications showed the highest expression levels whereas genomic deletions were correlated with samples exhibiting the lowest expression levels (S1 Fig).

Following these results, we decided to evaluate the relationship between CNV and the presence or absence of estrogen (ER), progesterone (PR) and epidermal growth hormone 2 (HER2) receptors. The absence of these receptors in BC is associated with a much more aggressive phenotype and an inability to use hormonal therapies to stop its development (Fig 1B). We observed a higher proportion of amplifications and gains of CNV in ER (-) and PR (-) samples than in ER (+) and PR (+) samples, respectively.

203 We evaluated BIRC6 CNV distribution according to chemotherapy and radiotherapy 204 treatments. Fig 1C shows that in samples from patients who received either of the two therapies, there was a greater amplification and gain percentage compared to those who did not.

207 After evaluation of the average age at which patients with the different CNVs were diagnosed, it became apparent that patients with higher CNVs tended to be diagnosed at a younger age than those who maintained diploidy or had deletions in BIRC6 gene (Fig 1D). We assessed 
210 the influence of CNV on two parameters that reflect prognosis: Nottingham Index and the

211 Neoplastic Histological Grade. It was observed that patients with amplifications had a higher

212 Nottingham Index than the rest of the conditions, thus implying worse prognosis (Fig 1E).

213 Furthermore, we found a higher proportion of patients with amplifications and gains of the

214 BIRC6 gene in patient samples with Histological Grade 3 (Fig 1E).

\subsection{BIRC6 expression and survival}

216 Breast cancer patient survival was evaluated using data of 1084 samples from TCGA

217 PanCancer Atlas. Patients were divided into two groups, high and low BIRC6 expression, and

218 survival was plotted for both groups. Survival time was significantly lower for patients with

219 higher BIRC6 expression levels (Fig 2A).

A

Survival

cohort: TCGA Pan-Cancer (PANCAN) $(n=1236)$

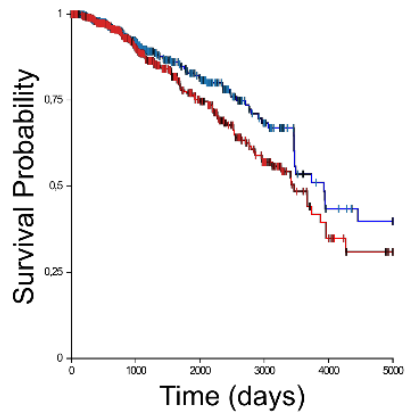

BIRC6 Expression

- Low Expression

-High Expression

B

Metastatic markers

cohort: TCGA Pan-Cancer (PANCAN) (n=1236)

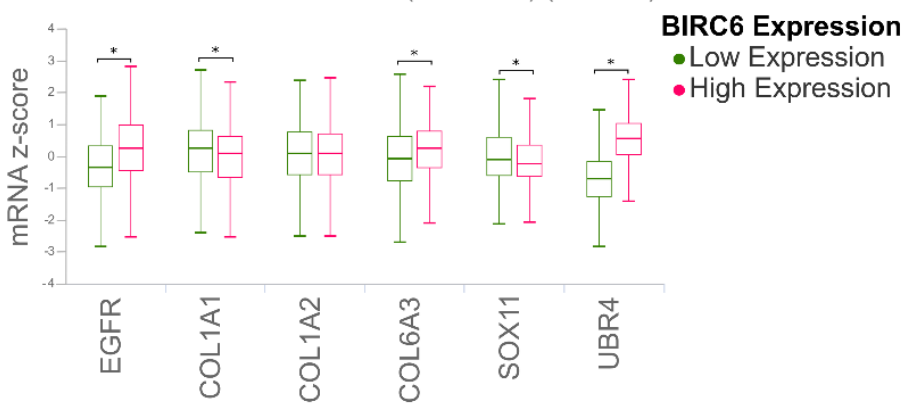

221 Fig 2. Survival and metastatic markers. (A) Survival plot. BC samples were filtered from TCGA Pan-

222 Cancer and the expression of BIRC6 was evaluated. In the analysis, the samples were divided into two 223 groups: high expression of BIRC6 and low expression of BIRC6 and survival were plotted for each 
group. T-test * p-value<0.05. (B) Metastatic markers expression. Boxplot of the expression (z-score) of common metastatic markers in condition of high and low BIRC6 expression. T-test * $p$-value $<0.01$.

\subsection{BIRC6 expression and pathways}

227 Differential expression of six general metastasis markers (EGFR, COL1A1, COL1A2, 228 COL6A3, SOX11 and UBR4) was determined in patients with low and high expression of BIRC6. EGFR, COL6A3 and UBR4 showed a significant increase in their expression levels in samples with high BIRC6 expression (Fig 2B). Furthermore, we observed a significant increase in LDHA and a significant decrease in PDH mRNA levels in samples with high expression of BIRC6 in accordance with the expected metabolic switch for tumor cells (S2 Fig and S1 Table). For a more systematic approach we conducted a Differential Expression Analysis (DEA) followed by a gene ontology study. Differential genes obtained in this analysis were divided in two output groups: overexpressed (Fig 3) and less expressed (S3 Fig) genes, both under conditions of high BIRC6 expression levels. Fig 3 summarizes biological processes, cellular components, molecular function and pathways in which overexpressed genes are involved. We found that genes involved in gene expression (nucleosome and

239 chromatin assembly and organization, protein-DNA complexes and DNA packaging) were 240 overexpressed in the analyzed conditions as well as those involved in actin and myosin 241 cytoskeleton signaling, epithelial adherence signaling and tight junction signaling. 


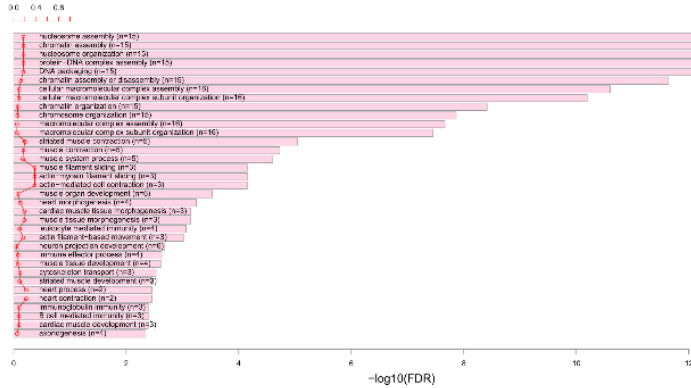

GO:Cellular Component

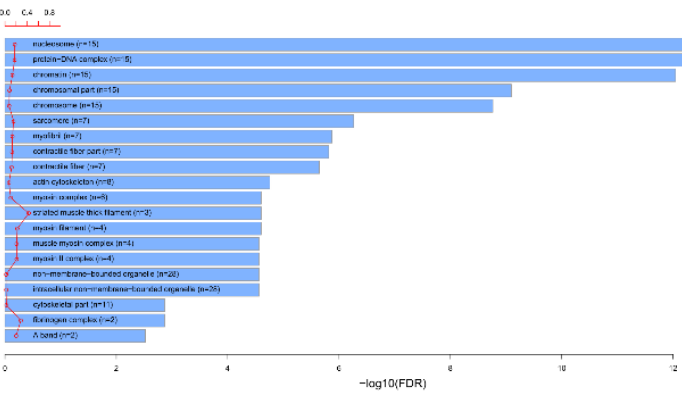

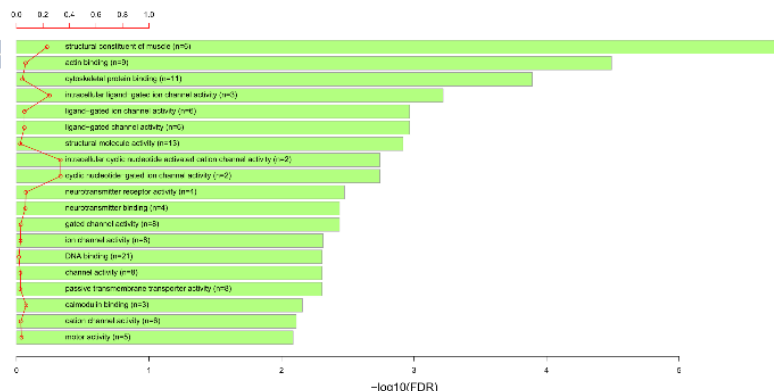

GO:Molecular Function

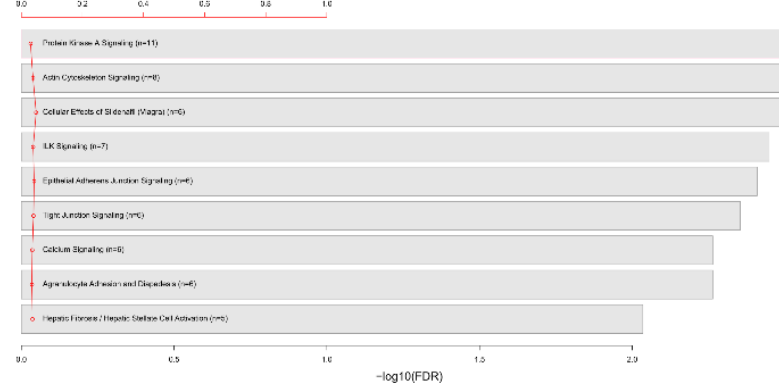

243 Fig 3. Gene ontology and over-represented pathways. Graphs show the canonical pathways 244 significantly over-represented (enriched) by the DEGs (differentially expressed genes) with the number 245 of genes for the main categories of the three ontologies (GO: biological process, GO: cellular 246 component, and GO: molecular function, respectively). The statistically significant canonical pathways 247 in the DEGs are listed according to their $p$-value corrected by FDR (- $\log 10)$ (colored bars) and the ratio 248 of the listed genes found in each pathway over the total number of genes in that pathway (ratio, red 249 line).

250 These results suggest that BIRC6 could play an important role in tumor homeostasis and metastasis development. For this reason, we decided to perform a deeper analysis on apoptosis, proliferation, angiogenesis, migration and focal adhesion pathways.

\section{i. Apoptosis}

254 We evaluated the expression of Bax, Bcl-2, Caspase 9, Caspase 3, Caspase 8, TP53, 255 Cytochrome C, NFKB1 and DIABLO under conditions of either low or high BIRC6 expression 
256 (Fig 4A). In addition, correlation between the expression of BIRC6 and the aforementioned 257 proteins was analyzed (Fig 4B) by grouping samples according to four criteria: normal tissue, 258 tumor tissue and positive or negative hormone receptor (HR + or HR-). The results showed 259 that those samples with high expression of BIRC6 had lower expression of Bax, Caspase 9, 260 Cytochrome C and DIABLO, and higher expression of $\mathrm{Bcl}-2$, Caspase 3, Caspase 8 and 261 NFKB1 when compared to samples with low BIRC6 expression. Furthermore, we observed 262 that there was no statistically significant difference in TP53 expression levels between both 263 groups (Fig 4B). Finally, we observed a differential correlation between samples of healthy 264 tissue and tumor tissue. 


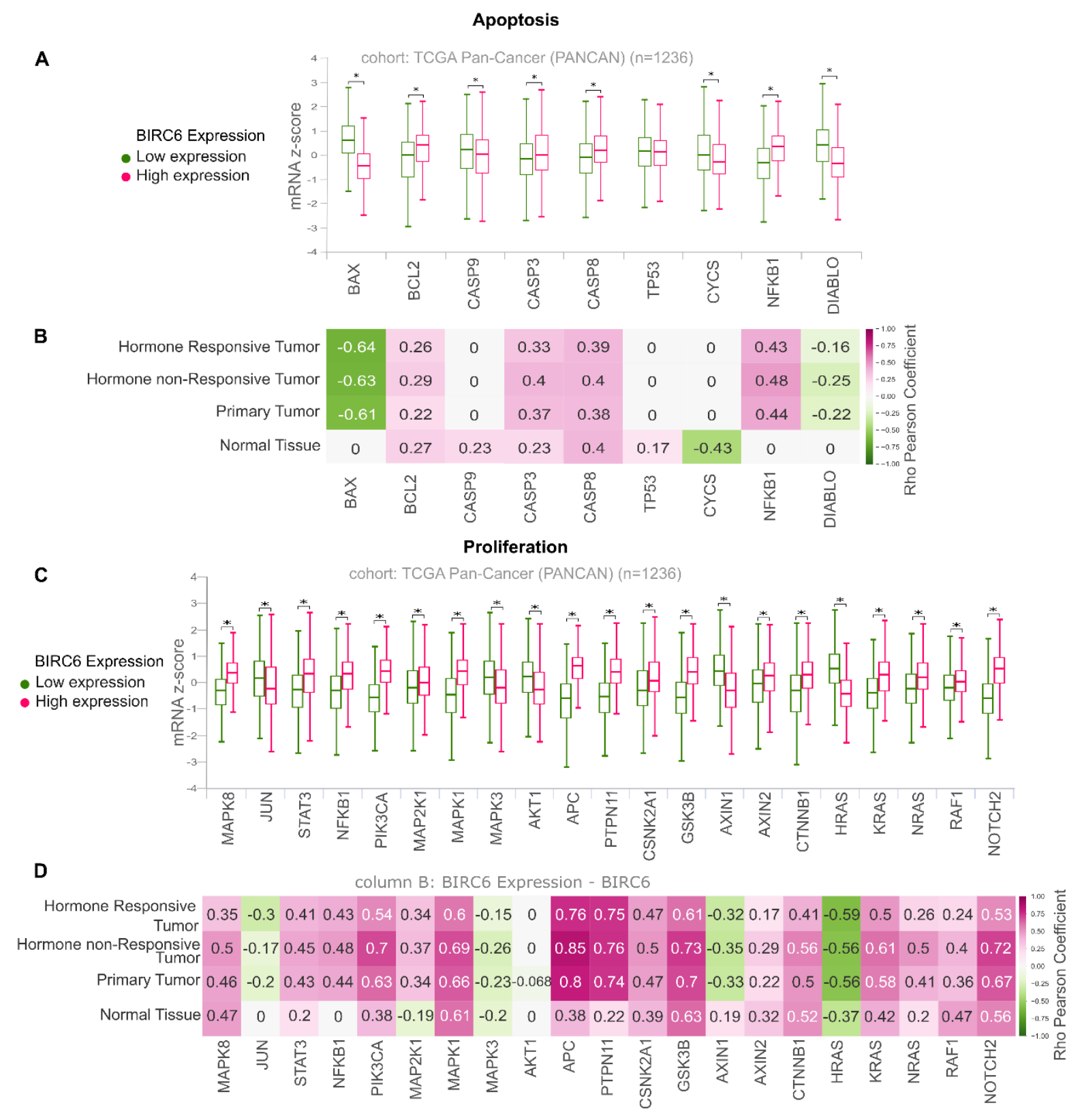

Fig 4. Transcriptomic analysis of apoptotic and proliferation pathways (TCGAPanCancer). (A)

267 Boxplot Of The Expression (z-score) of proteins involved in the apoptotic pathway in condition of high

268 and low BIRC6 expression. T-test *p-value $<0.01$. (B) Correlation between BIRC6 expression and 269 proteins involved in the apoptotic pathway separated in: normal tissue, primary tumor, hormone 270 responsive and hormone non responsive tumors. Numbers in cells indicate correlation pearson index 271 in statistically significant comparisons. (C) Boxplot of the expression (z-score) of proteins involved in 272 proliferative pathways in condition of high expression and low expression of BIRC6. T-test * $\mathrm{p}$ value $<0.01$. (D) Correlation between BIRC6 expression and proteins involved in proliferative pathways 
separated in normal tissue, primary tumor, hormone responsive and hormone non responsive tumors.

Numbers in cells indicate correlation pearson index in statistically significant comparisons.

\section{ii. Proliferation}

277 Another essential cellular process for tumor biology is proliferation, which is exacerbated in 278 cancer cells. Taking this into account, we repeated the previous analysis using proteins linked 279 to this process. We evaluated the expression of MAPK8, JUN, STAT3, NFkB1, PIK3CA, MAP2K1, MAPK1, MAPK3, AKT1, APC, PTPN11, CSNK2A1, GSK3B, AXIN1, AXIN2, CTNNB1, HRAS, KRAS, NRAS, RAF1 and NOTCH2 under conditions of low and high BIRC6 expression (Fig 4C). In addition, the correlation between the expression of these proteins and BIRC6 was analyzed in normal tissue, tumor tissue and positive or negative hormone receptors samples (Fig 4D). The results showed that those samples with high expression of BIRC6 have high expression of some proteins that promote proliferative pathways such as STAT3, PI3KCA, MAPKs and APC.

\section{iii. Metastatic cascade: Angiogenesis, Migration and Focal Adhesion}

288 The metastatic cascade consists of several steps: invasion, angiogenesis, surviving the 289 passage through the circulatory system, adhesion and anchorage in distant organs and, 290 finally, growth into micrometastases and, then, into consolidated metastases. We evaluated the correlation between BIRC6 overexpression and key regulators of the metastatic cascade.

292 Angiogenesis is the generation of new blood vessels from pre-existing ones, which allow tumor 293 cells to spread to distant organs. We assessed the expression levels of EGFR, IGF1R, INSR, 294 HIF1A, ARNT, ANGPT, TEK and VEGFA in conditions of both low and high BIRC6 expression 295 (Fig 5A). In addition, the correlation between the expression of these proteins and BIRC6 was 296 analyzed in samples of normal tissue, tumor tissue and positive or negative hormone receptors 297 (HR + or HR-). We found that those samples with high expression of BIRC6 have high expression levels of some angiogenesis promoters such as HIF1A and VEGFA (Fig 5B). 


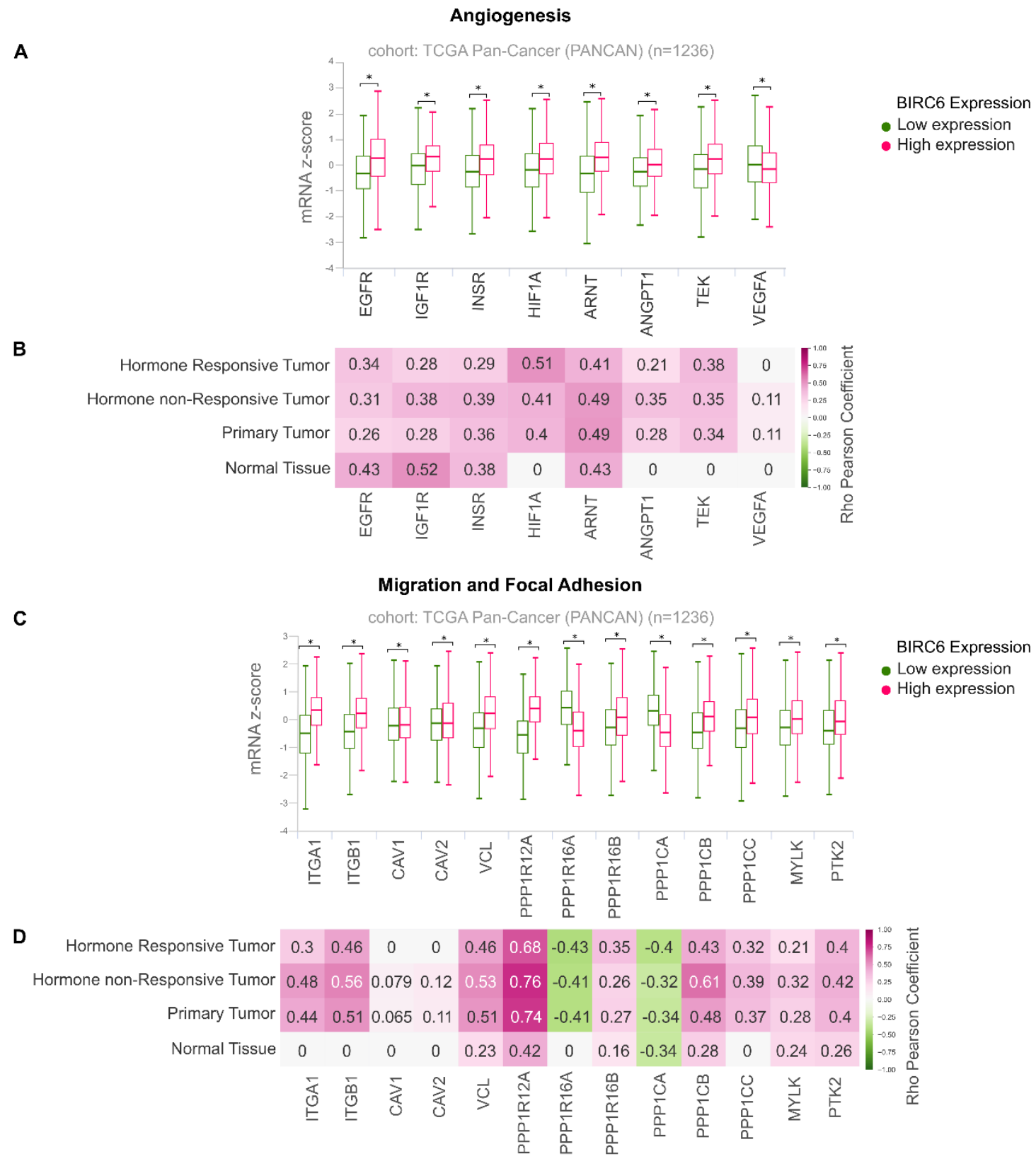

Fig 5. Transcriptomic analysis of pathways related to angiogenesis, migration and focal adhesion (TCGAPanCancer). (A) Boxplot Of The Expression (z-score) of proteins involved in angiogenesis in conditions of high and low expression of BIRC6. T-test ${ }^{*} \mathrm{p}$-value $<0.0$.

(B) Correlation between BIRC6 expression and proteins involved in the angiogenesis pathway separated in normal tissue, primary tumor, hormone responsive and hormone non responsive tumors. Numbers in cells indicate correlation pearson index in statistically significant comparisons. (C) Boxplot of the expression (z-score) of proteins involved in migration and focal adhesion related pathways in condition of high expression and proteins involved in migration and focal adhesion related pathways separated in normal 
tissue, primary tumor, hormone responsive and hormone non responsive tumors. Numbers in cells indicate correlation pearson index in statistically significant comparisons.

311 We also evaluated the role of BIRC6 on migration and adhesion processes, since both are

312 necessary for tumor cells to invade other tissues and colonize distant sites, thus generating

313 metastatic nodules. We evaluated the expression of ITGA1, ITGB1, CAV1, CAV2, VCL, 314 PPP1R12A, PPP1R16A, PPP1R16B, PPP1CA, PPP1CB, PPP1CC, MYLK and PTK2 under conditions of low and high BIRC6 expression (Fig 5C). We found that those samples with higher BIRC6 expression presented higher expression of some of the proteins that promote

317 migration such as VCL, PPP1R12A, PPP1CB, MYLK and of those involved in focal adhesions 318 like PTK2.

319 In addition, we see a distinctive behavior regarding the correlation of BIRC6 and the different proteins analyzed in tumor samples when compared to normal tissue, showing an increased correlation (Fig 5D). This implies a relationship between the expression of BIRC6 and different proteins involved in metastatic cascade. The complete study is presented in S2 Fig and S1 Table.

\section{Discussion}

325 Breast Cancer is the second leading cause of cancer death worldwide. With the emergence of resistance to conventional therapies arises a growing trend towards the design of new treatment schemes that employ specific molecular targets. Taking this into account, we set out to evaluate the role of BIRC6 in BC development and metastasis by genomic and transcriptomic approaches.

330 To begin the study, we analyzed BIRC6 expression in different tissues, and we found higher expression levels in samples from mammary carcinomas compared to those corresponding to healthy tissue. This could imply that an increase in the expression of BIRC6 could aid cells in evading the physiological mechanisms of homeostasis, and subsequently become carcinogenic $(7,9)$. 
335 We then assessed the correlation between different clinical attributes and the expression 336 levels of BIRC6 using databases that contained transcriptomic and genomic information of 337 patient samples.

338 The genomic analysis indicates a higher proportion of amplifications and gains in the number of copies of BIRC6 in ER- and PR- samples when compared to ER+ and PR+. The loss of hormone receptors results in tumors displaying much more aggressive phenotypes (46-51), thus suggesting a possible relationship between BIRC6 expression levels and tumor aggressiveness. We also compared the CNVs with the age of diagnosis and prognosis and

343 found that patients with amplifications or gains in BIRC6 copy number had a tendency to be 344 diagnosed at a younger age. Various studies have shown a correlation between BIRC6 expression and the patient's prognosis, as well as an involvement of BIRC6 in early stages of cancer development, for colorectal (6), prostate $(7,8)$, and ovarian cancer (9), among others. In the light of this, the relationship of BIRC6 CNV and neoplastic histological grade and the

348 Nottingham prognostic index were analyzed (52-54). We observed that those samples with 349 amplifications or gains showed a tendency to have worse prognosis and to be in more 350 advanced neoplastic degrees. This, together with the loss of the receptors, suggests that an increase in the number of BIRC6 copies could lead to early development and the generation of a more aggressive BC phenotype. To conclude the genomic part of the study, we decided to evaluate the CNV profile in patients who had received either chemotherapy or radiotherapy. It was reported for other types of cancer that BIRC6 could participate in the mechanisms of resistance to these therapies $(24,27)$. We found that in samples of patients who had received chemotherapy or radiotherapy there was a higher proportion of amplifications and gains in gene copy number. Since many signaling pathways are affected in tumor physiology, transcriptomic analysis of the main pathways involved were performed.

We evaluated the state of the different pathways under conditions of high and low BIRC6 expression and the correlation with the different proteins involved, in samples of hormone dependent and independent mammary carcinomas. Regarding apoptosis, gene selection was 
some kind of correlation in their expression, and also, regulating other points of the pathway that are not directly related. Furthermore, Jinyu Ren et al. reported that the BIRC6 gene is capable of regulating the P53 protein and the mitochondrial apoptotic pathway (15). We observed that the expression of anti-apoptotic genes such as BCL-2 and NFKB1 was increased in high BIRC6 expression samples and pro-apoptotic genes such as BAX, CASP9 and DIABLO were decreased. Despite some effectors with pro-apoptotic activity, like CASP3 and CASP8, correlated with BIRC6 expression, it is important to take into account that BIRC6 interacts directly with both of them exhibiting an inhibitory effect where the net balance results in apoptosis inhibition. Interestingly, despite previous reports in which a relationship between BIRC6 and TP53 was established in breast cancer (22), we found no differences in our transcriptomic analysis.

374 Another mechanism involved in tumor formation is the unregulated and excessive cell proliferation. It was demonstrated that BIRC6 inhibition decreases tumor cell growth in lung and prostate cancer $(8,24)$. In addition, our results demonstrate that BIRC6 overexpression may promote proliferative pathways like STAT3, PI3KCA, MAPKs and APC in BC. Tumor metastasis is a multistage process during which malignant cells spread from the primary tumor to non-contiguous organs. The steps that lead to metastasis can be summarized in a few events that are known as the metastatic cascade (55). Considering that metastatic spread affects the survival and prognosis of cancer patients, each of the events that are part of the metastatic cascade are attractive targets for developing therapeutic strategies in oncology. In this work we studied the correlation between BIRC6 expression and key regulators of the metastatic cascade. We observed BIRC6 overexpression correlated with high expression of different proteins involved in migration (such as MYLK), angiogenesis (HIF1A and VEGFA) and adhesion (PTK). Elevated levels of BIRC6 have been linked to cell growth and to some key steps in metastasis in different types of tumors. In lung cancer, BIRC6 overexpression was associated with tumor progression, cell growth, colony formation, migration and invasion as well as with patient metastasis stage (3). In addition, in prostate cancer, the reduction of BIRC6 expression decreases cancer cell viability and proliferation 
$391(7,8)$. We propose that BIRC6 could be involved in tumor progression and metastatic cascade 392 in BC. However, further experiments are needed to explore our proposal in vivo.

\section{Conclusion}

394 In this work, we proposed an integrative study based on genomic and transcriptomic analyses, with the aim of characterizing the role of the apoptosis inhibitor BIRC6 in Breast Cancer.

396 Genomic results show that a higher copy number of BIRC6 gene correlates with more 397 aggressive phenotypes and worse prognosis markers. Transcriptomic studies show that 398 BIRC6 is overexpressed in tumor cells and these levels strongly correlate with an antiapoptotic 399 / proliferative profile. Finally, BIRC6 could play a role in the activation of signaling pathways involved in metastasis.

401 Altogether our collected data suggest that BIRC6 plays an important role in tumor homeostasis and can be related with the different stages of the metastatic cascade such as angiogenesis, migration and adhesion in BC (Fig 6).



405 Fig 6. Schematic representation of metastatic cascade. Steps of metastasis are represented in three 406 groups: principal pathways in tumor microenvironment (1), pathways involved in tumor cell spreading 
(2) and principal pathways in metastatic target organ (3). Principal genes with BIRC6 positive correlation are indicated in green whilst negatively correlated are indicated in red. (Created using BioRender.com).

409 The results of in silico analyses presented here make it possible to postulate the inhibitor of 410 apoptosis BIRC6 as an interesting molecular target for the development of specific therapies 411 for the reduction of tumor progression and metastasis in patients with Breast Cancer.

\section{Acknowledgements}

413 We thank the Agencia Nacional de Promoción Científica y Tecnológica (ANPCyT), the 414 Consejo Nacional de Investigaciones Científicas y Técnicas (CONICET), National University 415 of La Plata (UNLP) and National University of Quilmes (UNQ).

\section{References}

417 1. Turashvili G, Brogi E. Tumor Heterogeneity in Breast Cancer. Front Med. 8 de diciembre de 2017;4:227.

419 2. Kim RS, Avivar-Valderas A, Estrada Y, Bragado P, Sosa MS, Aguirre-Ghiso JA, et al. 420 Dormancy Signatures and Metastasis in Estrogen Receptor Positive and Negative Breast Cancer. Minna JD, editor. PLoS ONE. 18 de abril de 2012;7(4):e35569.

3. Fares J, Fares MY, Khachfe HH, Salhab HA, Fares Y. Molecular principles of metastasis: a hallmark of cancer revisited. Signal Transduct Target Ther. diciembre de 2020;5(1):28.

4. Silke J, Ekert PG, Day CL, Hawkins CJ, Baca M, Chew J, et al. Direct inhibition of caspase 3 is dispensable for the anti-apoptotic activity of XIAP. EMBO J. junio de 2001;20(12):3114-23.

5. Mohamed MS, Bishr MK, Almutairi FM, Ali AG. Inhibitors of apoptosis: clinical implications in cancer. Apoptosis. diciembre de 2017;22(12):1487-509.

6. Hu T, Weng S, Tang W, Xe R, Chen S, Cai G, et al. Overexpression of BIRC6 is a predictor of prognosis for colorectal cancer. PLoS ONE. mayo de 2015;10(5).

7. Low CG, Luk ISU, Lin D, Fazli L, Yang K, Xu Y, et al. BIRC6 Protein, an Inhibitor of 
2013;8(2).

434 8. Iris Luk SU, Xue H, Cheng H, Lin D, Gout PW, Fazli L, et al. The BIRC6 gene as a novel target for therapy of prostate cancer: Dual targeting of inhibitors of apoptosis. Oncotarget. 2014;5(16):6896-908.

9. Wang L, Chen YJ, Hou J, Wang YY, Tang WQ, Shen XZ, et al. Expression and clinical significance of BIRC6 in human epithelial ovarian cancer. Tumor Biol. 2014;35(5):4891-

6.

10. Liang J, Zhao W, Tong $\mathrm{P}$, Li $\mathrm{P}$, Zhao $\mathrm{Y}$, Li H, et al. Comprehensive molecular characterization of inhibitors of apoptosis proteins (IAPs) for therapeutic targeting in cancer. BMC Med Genomics. diciembre de 2020;13(1):7.

11. Bartke T, Pohl C, Pyrowolakis G, Jentsch S. Dual Role of BRUCE as an Antiapoptotic IAP and a Chimeric E2/E3 Ubiquitin Ligase. Mol Cell. junio de 2004;14(6):801-11.

12. Sekine K, Hao Y, Suzuki Y, Takahashi R, Tsuruo T, Naito M. HtrA2 cleaves Apollon and induces cell death by IAP-binding motif in Apollon-deficient cells. Biochem Biophys Res Commun. abril de 2005;330(1):279-85.

13. Hao $Y$, Sekine K, Kawabata A, Nakamura H, Ishioka $T$, Ohata H, et al. Apollon ubiquitinates SMAC and caspase-9, and has an essential cytoprotection function. Nat Cell Biol. septiembre de 2004;6(9):849-60.

14. Qiu XB, Goldberg AL. The membrane-associated inhibitor of apoptosis protein, BRUCE/Apollon, antagonizes both the precursor and mature forms of smac and caspase-9. J Biol Chem. enero de 2005;280(1):174-82.

15. Ren J, Shi M, Liu R, Yang QH, Johnson T, Skarnes WC, et al. The Birc6 (Bruce) gene regulates p53 and the mitochondrial pathway of apoptosis and is essential for mouse embryonic development. Proc Natl Acad Sci U S A. enero de 2005;102(3):565-70.

16. Hauser HP, Bardroff M, Pyrowolakis G, Jentsch S. A giant ubiquitin-conjugating enzyme related to IAP apoptosis inhibitors. J Cell Biol. junio de 1998;141(6):1415-22. 
18. Ikeda F. The anti-apoptotic ubiquitin conjugating enzyme BIRC6/BRUCE regulates autophagosome-lysosome fusion. Autophagy. 3 de julio de 2018;14(7):1283-4.

19. Jia R, Bonifacino JS. Negative regulation of autophagy by uba6-birc6-mediated ubiquitination of Ic3. eLife. noviembre de 2019;8.

20. Ki WS, Choi J, Yu KH, Sang JL, Kim HJ, Soo HL, et al. Overexpression of Apollon, an antiapoptotic protein, is associated with poor prognosis in childhood de novo acute myeloid leukemia. Clin Cancer Res. septiembre de 2007;13(17):5109-14.

21. Bianchini M, Levy E, Zucchini C, Pinski V, Macagno C, De Sanctis P, et al. Comparative study of gene expression by cDNA microarray in human colorectal cancer tissues and normal mucosa. Int J Oncol (Internet). 1 de julio de 2006 (citado 1 de marzo de 2021); Disponible en: http://www.spandidos-publications.com/10.3892/ijo.29.1.83

22. Lopergolo A, Pennati M, Gandellini P, Orlotti NI, Poma P, Daidone MG, et al. Apollon gene silencing induces apoptosis in breast cancer cells through p53 stabilisation and caspase-3 activation. Br J Cancer. marzo de 2009;100(5):739-46.

23. Chen Z, Naito M, Hori S, Mashima T, Yamori T, Tsuruo T. A human IAP-family gene, Apollon, expressed in human brain cancer cells. Biochem Biophys Res Commun. noviembre de 1999;264(3):847-54.

24. Dong X, Lin D, Low C, Vucic EA, English JC, Yee J, et al. Elevated Expression of BIRC6 Protein in Non-Small-Cell Lung Cancers is Associated with Cancer Recurrence and Chemoresistance. J Thorac Oncol. febrero de 2013;8(2):161-70.

25. Chu L, Gu J, Sun L, Qian Q, Qian C, Liu X. Oncolytic adenovirus-mediated shRNA against Apollon inhibits tumor cell growth and enhances antitumor effect of 5-fluorouracil. Gene Ther. abril de 2008;15(7):484-94.

26. Van Houdt WJ, Emmink BL, Pham TV, Piersma SR, Verheem A, Vries RG, et al. Comparative proteomics of colon cancer stem cells and differentiated tumor cells identifies BIRC6 as a potential therapeutic target. Mol Cell Proteomics. diciembre de 2011;10(12):1-11.

27. Ismail EAR, Mahmoud HM, Tawfik LM, Habashy DM, Adly AAM, El-Sherif NH, et al. 
BIRC6/Apollon gene expression in childhood acute leukemia: Impact on therapeutic response and prognosis. Eur J Haematol. febrero de 2012;88(2):118-27.

28. Lamers F, Schild L, Koster J, Speleman F, Øra I, Westerhout EM, et al. Identification of BIRC6 as a novel intervention target for neuroblastoma therapy. BMC Cancer. julio de 2012;12:285.

29. Connolly K, Mitter R, Muir M, Jodrell D, Guichard S. Stable XIAP knockdown clones of HCT116 colon cancer cells are more sensitive to TRAIL, taxanes and irradiation in vitro. Cancer Chemother Pharmacol. julio de 2009;64(2):307-16.

30. Holt SV, Brookes KE, Dive C, Makin GWJ. Down-regulation of XIAP by AEG35156 in paediatric tumour cells induces apoptosis and sensitises cells to cytotoxic agents. Oncol Rep. abril de 2011;25(4):1177-81.

500

31. Salehi S, Jafarian AH, Montazer M, Moghbeli M, Forghanifard MM. BRUCE Protein, New Marker for Targeted Therapy of Gastric Carcinoma. J Gastrointest Cancer. junio de $2017 ; 48(2): 151-5$.

32. Curtis C, Shah SP, Chin SF, Turashvili G, Rueda OM, Dunning MJ, et al. The genomic 504 505 and transcriptomic architecture of 2,000 breast tumours reveals novel subgroups. Nature. junio de 2012;486(7403):346-52.

33. Pereira B, Chin SF, Rueda OM, Vollan HKM, Provenzano E, Bardwell HA, et al. The 508 somatic mutation profiles of 2,433 breast cancers refines their genomic and transcriptomic landscapes. Nat Commun. mayo de 2016;7.

34. Goldman MJ, Craft B, Hastie M, Repečka K, McDade F, Kamath A, et al. Visualizing and interpreting cancer genomics data via the Xena platform. Vol. 38, Nature Biotechnology.

35. Sanchez-Vega F, Mina M, Armenia J, Chatila WK, Luna A, La KC, et al. Oncogenic Signaling Pathways in The Cancer Genome Atlas. Cell. abril de 2018;173(2):321337.e10.

36. Hoadley KA, Yau C, Hinoue T, Wolf DM, Lazar AJ, Drill E, et al. Cell-of-Origin Patterns Dominate the Molecular Classification of 10,000 Tumors from 33 Types of Cancer. Cell. 
abril de 2018;173(2):291-304.e6.

518 37. Bailey MH, Tokheim C, Porta-Pardo E, Sengupta S, Bertrand D, Weerasinghe A, et al. Comprehensive Characterization of Cancer Driver Genes and Mutations. Cell. abril de 2018;173(2):371-385.e18.

38. Grossman RL, Heath AP, Ferretti V, Varmus HE, Lowy DR, Kibbe WA, et al. Toward a Shared Vision for Cancer Genomic Data. N Engl J Med. septiembre de 2016;375(12):1109-12.

39. Cerami E, Gao J, Dogrusoz U, Gross BE, Sumer SO, Aksoy BA, et al. The cBio Cancer Genomics Portal: An open platform for exploring multidimensional cancer genomics data. Cancer Discov. mayo de 2012;2(5):401-4.

40. Gao J, Aksoy BA, Dogrusoz U, Dresdner G, Gross B, Sumer SO, et al. Integrative analysis of complex cancer genomics and clinical profiles using the cBioPortal. Sci Signal. abril de 2013;6(269).

41. Li B, Dewey CN. RSEM: Accurate transcript quantification from RNA-Seq data with or without a reference genome. BMC Bioinformatics. agosto de 2011;12.

42. Colaprico A, Silva TC, Olsen C, Garofano L, Cava C, Garolini D, et al. TCGAbiolinks: an R/Bioconductor package for integrative analysis of TCGA data. Nucleic Acids Res. 5 de mayo de 2016;44(8):e71-e71.

43. Silva TC, Colaprico A, Olsen C, D'Angelo F, Bontempi G, Ceccarelli M, et al. TCGA Workflow: Analyze cancer genomics and epigenomics data using Bioconductor packages. F1000Research. 28 de diciembre de 2016;5:1542.

44. Mounir M, Lucchetta M, Silva TC, Olsen C, Bontempi $G$, Chen $X$, et al. New functionalities in the TCGAbiolinks package for the study and integration of cancer data from GDC and GTEx. Wang E, editor. PLOS Comput Biol. 5 de marzo de 2019;15(3):e1006701.

45. R Core Team. R: A Language and Environment for Statistical Computing. R Foundation for Statistical Computing: R Foundation for Statistical Computing; Vienna Austria.; 2020.

46. Chacón RD, Costanzo MV. Triple-negative breast cancer. Vol. 12, Breast Cancer 
Research. BioMed Central Ltd.; 2010. S3 p.

546

547

548

549

550

551

552

553

554

555

556

557

558

559

560

561

562

563

564

565

566

47. The Cancer Genome Atlas Network. Comprehensive molecular portraits of human breast tumours. Nature. 2012;490:61-70.

48. Perou CM, Sørile T, Eisen MB, Van De Rijn M, Jeffrey SS, Ress CA, et al. Molecular portraits of human breast tumours. Nature. agosto de 2000;406(6797):747-52.

49. Carey L, Winer E, Viale G, Cameron D, Gianni L. Triple-negative breast cancer: Disease entity or title of convenience? Vol. 7, Nature Reviews Clinical Oncology. Nat Rev Clin Oncol; 2010. 683-692 p.

50. Foulkes WD, Smith IE, Reis-Filho JS. Triple-Negative Breast Cancer. N Engl J Med. noviembre de 2010;363(20):1938-48.

51. Dawson SJ, Provenzano E, Caldas C. Triple negative breast cancers: Clinical and prognostic implications. Eur J Cancer. septiembre de 2009;45(SUPPL. 1):27-40.

52. Al jarroudi O, Zaimi A, Brahmi SA, Afqir S. Nottingham prognostic index is an applicable prognostic tool in non-metastatic triple-negative breast cancer. Asian Pac J Cancer Prev. enero de 2019;20(1):59-63.

53. Rakha EA, Reis-Filho JS, Baehner F, Dabbs DJ, Decker T, Eusebi V, et al. Breast cancer prognostic classification in the molecular era: The role of histological grade. Vol. 12, Breast Cancer Research. BioMed Central; 2010. 207 p.

54. Lee AHS, Ellis IO. The Nottingham prognostic index for invasive carcinoma of the breast. Vol. 14, Pathology and Oncology Research. Pathol Oncol Res; 2008. 113-115 p.

55. Welch DR, Hurst DR. Defining the Hallmarks of Metastasis. Cancer Res. 15 de junio de 2019;79(12):3011-27. 